\title{
Correlation $\bar{v}_{p}-\sigma-\chi$ in the fast neutron range via integral information
}

\author{
Dimitri Rochman ${ }^{1}$, Eric Bauge ${ }^{2,}$, Alexander Vasiliev ${ }^{1}$, and Hakim Ferroukhi ${ }^{1}$ \\ ${ }^{1}$ Laboratory for Reactor Physics Systems Behaviour, Paul Scherrer Institut, Villigen, Switzerland \\ ${ }^{2}$ CEA, DAM, DIF, 91297 Arpajon cedex, France
}

Received: 18 January 2017 / Received in final form: 20 March 2017 / Accepted: 20 March 2017

\begin{abstract}
This paper presents a Bayesian approach based on integral experiments to create correlations which do not appear with differential data. Some quantities such as the fission cross section $(\sigma)$, neutron multiplicity $\left(\bar{v}_{p}\right)$, neutron spectra $(\chi)$, etc. are usually neither modeled together nor measured in coincidence, thus there is no correlation matrices in evaluated nuclear data libraries. One can nevertheless use the information from integral experiments such as fast criticality-safety benchmarks to correlate such quantities for possible inclusion in nuclear data libraries. A simple Bayesian set of equations is presented with random nuclear data, similarly to the usual methods applied with differential data. An example for ${ }^{239} \mathrm{Pu}$ is proposed.
\end{abstract}

\section{Introduction}

Covariance information for basic nuclear data quantities is nowadays necessary for various types of nuclear applications. Many simulation tools are capable of using such matrices to propagate nuclear data uncertainties on final quantities, with either perturbation theories [1-6], or Monte Carlo sampling [3,7-13]. These results can for instance be used for the review procedure of new facilities, or during the safety assessment of new reactor core designs [14]. The origin of such covariance information can be found in various nuclear data libraries, such as ENDF/ B-VII [15,16], JENDL-4.0 [17] or TENDL [18], and the production techniques can widely vary, see for instance references [19,20,22-26].

All the methods applied to experimental observables (measured cross sections, angular and energy distributions) provide nominal values, with uncertainties and correlations, for instance in terms of covariance matrices between incident neutron energies for a specific reaction channel, e.g. fission, or capture. With the help of nuclear reaction models, cross-channel covariance matrices can be obtained, for instance between the fission and the capture cross sections. Alternatively, the methods solely based on model parameters, such as in references [19,20], also lead to similar matrices.

One of the drawbacks of the existing approaches is that no correlations are proposed between quantities which are not simultaneously measured, or calculated within a

\footnotetext{
* e-mail: dimitri-alexandre.rochman@psi.ch
}

unique theoretical model. For instance, there is currently no measurements providing in coincidence a fission rate (related to the fission cross section $\sigma(\mathrm{n}, \mathrm{f})$ ) and the number of emitted prompt neutron per fission (so-called nubar, or $\bar{v}_{p}$ ). It can nevertheless be noted that some efforts are spent to obtain such data, as presented in reference [21]. In the following $\sigma(\mathrm{n}, \mathrm{f})$ and $\bar{v}_{p}$ will simply be noted $\sigma$ and $\nu$, respectively. Similarly, the reaction models separately calculate cross sections and prompt neutron emitted spectra $(\chi)$ as independent quantities. Therefore, no theoretical correlation matrix can be obtained between $\sigma, v$ and $\chi$. One may argue that if quantities are not measured or calculated together, there is no correlation between them. Nonetheless correlations and uncertainties are valid within the applied method and can therefore be calculated in a well-defined framework. With the example of $\sigma-v-\chi$, if there are no differential evidence of such correlation (at the level of the observables, i.e. cross sections and emitted neutrons), there are nevertheless indications from integral measurements. It is also important to realize that missing correlation matrices in evaluated files correspond to zero correlations. Such correlations can affect uncertainties for integral quantities, as uncorrelated nuclear data can be either independently sampled (for Monte Carlo uncertainty propagation), or can lead to simplified covariance matrices (for sensitivity \& perturbation methods). Moreover, these zero correlation matrices are not visible and users are often not aware of the possible impact of these hidden correlations.

This paper proposes to evaluate correlation matrices between $\sigma, v$ and $\chi$ in the fast neutron range using integral information based on a simple Bayesian method, as 
presented in references [20,25]. As mentioned, such correlation matrices are not provided in nuclear data libraries, as they are in most of the cases based on differential measurements. But the use of integral benchmarks, such as fast criticality-safety benchmarks [27] can provide such values, as presented in Section 2. Examples for the ${ }^{239} \mathrm{Pu}$ fission cross section $(\sigma)$, its $v$ and $\chi$ will be presented in Section 3 for so-called fast benchmarks. Finally, an overview of the correlation matrices will be presented in Section 4, with possible other applications.

\section{Correlation from integral benchmarks}

As mentioned in the introduction, correlations between some quantities are not provided in libraries, unfortunately giving the presumption to the nuclear data users that they do not exist. One of the most important example concerns the fission cross section and $v$ for a given nucleus. Assuming that such quantities are estimated with an uncertainty and that design quantities are fixed, there is a small probability that their true values are equal to their mean estimates plus one standard deviation, for both of them at the same time. It is however more likely that from our simulation capabilities, their true values are anti-correlated: an estimated high fission cross section is probably to be compensated by a low $v$ (since the source term in the Boltzmann equation takes the form of $\sigma \times v$ ). A similar argument can be applied for the fission cross section and the hardness of $\chi$ (how high is the mean outgoing neutron energy, which drives the energy distribution of the fission neutrons).

This is the central point of this work: using numerical simulations based on these three quantities to extract their correlation matrices (e.g. with the Boltzmann transport equation). Derived integral quantities are numerous and easily calculated, and they can be compared to experimental integral data. In the present context, we will limit ourselves to criticality benchmarks with the calculation of the multiplication factor $k_{\text {eff }}$, for instance calculated with the neutron transport code MCNP [28]. In first approximations, $k_{\text {eff }}$ is proportional to the product of $v$ and $\sigma$, thus these two quantities are anti-correlated for a fixed $k_{\text {eff }}$ value.

The general proposed method is as follows. Within the assumptions that $\nu, \sigma$ and $\chi$ are not perfectly known and can be represented by independent probability distributions (prior assumption), it is possible to generate random realizations, and then to calculate random $k_{\text {eff }}$ values for a specific system definition (geometry, content, etc.). Such random $k_{\text {eff }}$ values can then be compared to a specific measurement of $k_{\text {eff }}$ with its uncertainty (called $k_{\exp } \pm \Delta \mathrm{k}$ ), using for instance a simplified chi-2 definition. The correlation between $v$ and $\sigma$ (and $\chi$ ) can then be obtained using weights and the usual covariance definitions (leading to posterior probabilities for the 3 quantities of interest).

In the following, an example is given for $v$ and $\sigma$. Any of these two quantities can also be replaced by $\chi$.

- Independent probability distributions (prior pdf): the calculation of these pdf is based on the sampling of model parameters (as in the TMC and BFMC methods $[19,20]$ ). Model parameters are sampled a large number of times (with the index $i=1, \ldots, n$ ) to generate full cross sections and other nuclear data quantities for ${ }^{239} \mathrm{Pu}$ from 0 to $20 \mathrm{MeV}$. The T6 system [18] is used to generate so-called random ENDF-6 and ACE files, containing all necessary random nuclear data. Such random files for ${ }^{239} \mathrm{Pu}$ can be found in the TENDL-2014 library [29]. As mentioned, in the T6 system, the $v$ values (as a function of the incident neutron energy) are independently calculated from $\sigma$, for all incident energies.

- The systems are fast criticality benchmarks. The advantage of these benchmarks is that their $k_{\text {eff }}$ is highly sensitive to the fast neutron range, thus avoiding the complications linked to the resolved and unresolved resonance range. MCNP6 is used to calculate $k_{\text {eff }, i}$ for the specific random ACE file $i$ for ${ }^{239} \mathrm{Pu}$.

- The comparison between $n$ random calculated and experimental $k_{\text {eff, } i=1 \ldots, n}$ is performed with the chi- $2 Q_{i}$ values and associated weights $w_{i}$ (here, chi-2 is called $Q_{i}$ to differentiate it from the neutron spectra $\chi$ ):

$$
\begin{aligned}
Q_{i} & =\left(\frac{k_{\mathrm{eff}, i}-k_{\text {exp }}}{\Delta k}\right)^{2}, \\
w_{i} & =\exp \left(-\frac{Q_{i}}{2}\right) .
\end{aligned}
$$

Such formulation can easily be linked to the usual Bayesian likelihood.

- Such weights are then assigned to the corresponding $v_{i}$ and $\sigma_{i}$ which lead to $k_{\text {eff }, i}$. The final quantity for a specific benchmark consists of a matrix containing $\left[v_{i}, \sigma_{i}, w_{i}\right]$ for $i=1, \ldots, n$. In the following, $n=10,000$. Figure 1 presents the distribution of $v$ and $\sigma$ with distinct colors being proportional to $w_{i}$ : red for small $w_{i}$ and black for $w_{i} \simeq 1$. The $w_{i}$ are obtained using the pmf1 benchmark (or Jezebel). A simple spline function gives an idea of the correlation between $v$ and $\sigma$ for their specific energies.

Each of the random ENDF-6 files in this work contains unique nuclear data realizations based on the sampling of the model parameters. They have already been used and characterized in previous work, see for instance references $[2,3,26]$. All possible nuclear data are varied, such as cross sections, angular distributions, emitted spectra and multiplicities. Therefore, the observed spread of $k_{\text {eff }}$ based is the reflection of all varied quantities. Still, if one of them (e.g. the fission cross section at a specific energy) has a dominant impact on $k_{\text {eff }}$, there will be a correlation with $k_{\text {eff }}$, independently of the variations of the other variables. Such correlation can be observed as long as the nuclear data of interest has a significant impact, somewhat not overshadowed by the action of the other variations. Such correlation factors are already used in reactor physics to order important input factors and quantify their impacts. They are directly related to the importance factors $R^{2}$, see for instance references $[5,30]$. The $R^{2}$ (or the correlation $\rho$ ) are very similar to the sensitivity vectors obtained in perturbation methods, but they also take into account the internal energy-energy correlation (for instance the fission cross section energy-energy correlation). Thus, correlations between input variables and the output might be found in a specific energy range, even if there is no sensitivity for this 

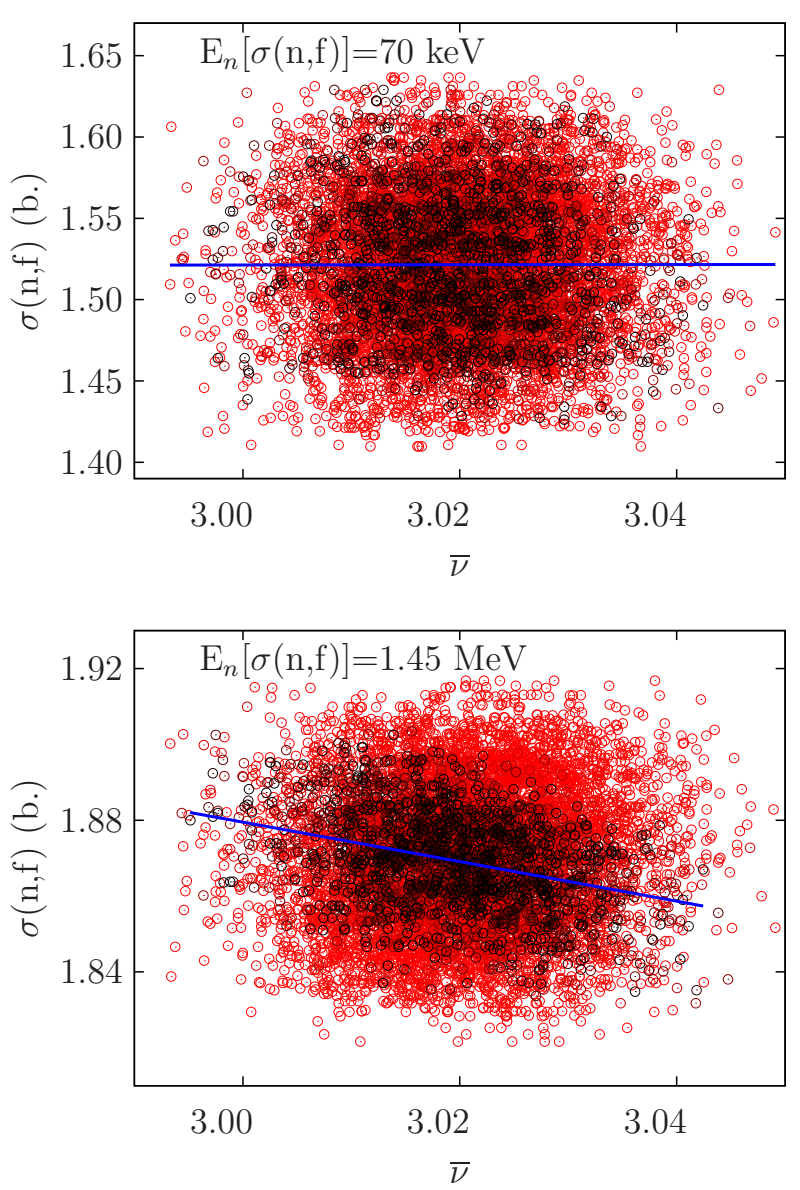

Fig. 1. Example of correlations between $v$ and $\sigma$ for two different incident neutron energies for the fission cross section. The incident neutron energy for $v$ is $900 \mathrm{keV}$ in both cases. The considered criticality benchmark is pmf1. 10000 samples are presented; the distinct colors are proportional to $w_{i}$ : red for small $w_{i}$ and black for $w_{i} \simeq 1$.

energy range, because of the energy-energy correlation with an energy region where the sensitivity is not negligible. This remark is important for the understanding of the following results, where some non-zero correlation values might be found for incident energies where the considered benchmark has a very small neutron population.

The correlation $\rho\left(E_{v}, E_{\sigma}\right)$ can be calculated for specific values of the incident neutron energies for $\nu\left(E_{v}\right)$ and $\sigma\left(E_{\sigma}\right)$. In the following, $\rho\left(E_{v}, E_{\sigma}\right), v\left(E_{v}\right)$ and $\sigma\left(E_{\sigma}\right)$ will be noted $\rho, v$ and $\sigma$ for simplicity. Considering the vector $\left[v_{i}, \sigma_{i}, w_{i}\right]$ with $n=10000$, $\rho$ can be calculated as follows. Using the definition of weighted averages:

$$
\left\{\begin{array}{l}
\omega=\sum_{i}^{n} w_{i} \\
\omega_{v}=\sum_{i}^{n} w_{i} \cdot v_{i} / \omega \\
\omega_{\sigma}=\sum_{i}^{n} w_{i} \cdot \sigma_{i} / \omega
\end{array}\right.
$$

and the definition of the weighted covariance factors:

$$
\left\{\begin{array}{l}
\operatorname{cov}_{\nu}=\sum_{i}^{n}\left[\left(v_{i}-\omega_{\nu}\right)^{2} \cdot w_{i}\right] / \omega \\
\operatorname{cov}_{\sigma}=\sum_{i}^{n}\left[\left(\sigma_{i}-\omega_{\sigma}\right)^{2} \cdot w_{i}\right] / \omega \\
\operatorname{cov}_{\sigma \nu}=\sum_{i}^{n}\left[\left(\sigma_{i}-\omega_{\sigma}\right) \cdot\left(v_{i}-\omega_{\nu}\right) \cdot w_{i}\right] / \omega
\end{array},\right.
$$

the correlation $\rho$ between $v$ and $\sigma$ is given by

$$
\rho=\frac{\operatorname{cov}_{\sigma v}}{\sqrt{\operatorname{cov}_{\nu} \cdot \operatorname{cov}_{\sigma}}} .
$$

Such correlation $\rho$ can be obtained for different $E_{\sigma}$ and $E_{v}$, thus defining a full correlation matrix. In the example of Figure 1 , the correlations are -0.10 and -0.34 for the two different energies of the fission cross section, $70 \mathrm{keV}$ and $1.45 \mathrm{MeV}$, respectively (the energy of $v$ is the same and equal to $900 \mathrm{keV}$ ). In the case of $\chi$, two energy grids are considered: the incident neutron energy and the outgoing neutron energy. The proposed set of equations can be applied to $\chi$ for a specific incident energy (for instance a incident neutron with an energy of $750 \mathrm{keV}$ ) and $E$ represents the outgoing neutron energy.

With these basis, correlation matrices can now be calculated for ${ }^{239} \mathrm{Pu}$, given a set of specific benchmarks. In the following, the statistical uncertainty of the $k_{\text {eff }}$ calculation performed with MCNP6 is in the order of $20 \mathrm{pcm}$.

A similar approach was applied in reference [31] where correlations between the inelastic cross sections of the $\mathrm{Cu}$ isotopes and a shielding benchmarks were obtained. In this reference, the correlation was not based on a weighting approach, but rather on a simple accept/reject method.

As a final remark, the statistical significance of the calculated correlation can be assessed as in reference [32] using the Student's t-test:

$$
|t|=\rho \times \sqrt{\frac{n-2}{1-\rho^{2}}},
$$

with the degree of freedom equal to $n-2$. The $t$-value is evaluated by the usual $t$-distribution: with $n=10,000$, a significance level of $95 \%$ is achieved for $|\rho|>1.6 \%$. In the case of $99.9 \%,|\rho|>3.1 \%$.

\section{Application to ${ }^{239} \mathrm{Pu}$}

The isotope ${ }^{239} \mathrm{Pu}$ presents several advantages from the nuclear data point of view. Its fission cross section, $v$ and $\chi$ are relatively well known in the fast neutron range (generally above a few tens of $\mathrm{keV}$ ) and it is commonly used for criticality benchmarks. Additionally, the production of random ENDF-6 and ACE files is relatively fast compared to other actinides, which allows to reach $n=10000$ without a large computer facility. Many criticality-safety benchmarks are highly sensitive to ${ }^{239} \mathrm{Pu}$ cross sections, namely all the "plutonium metal fast" 


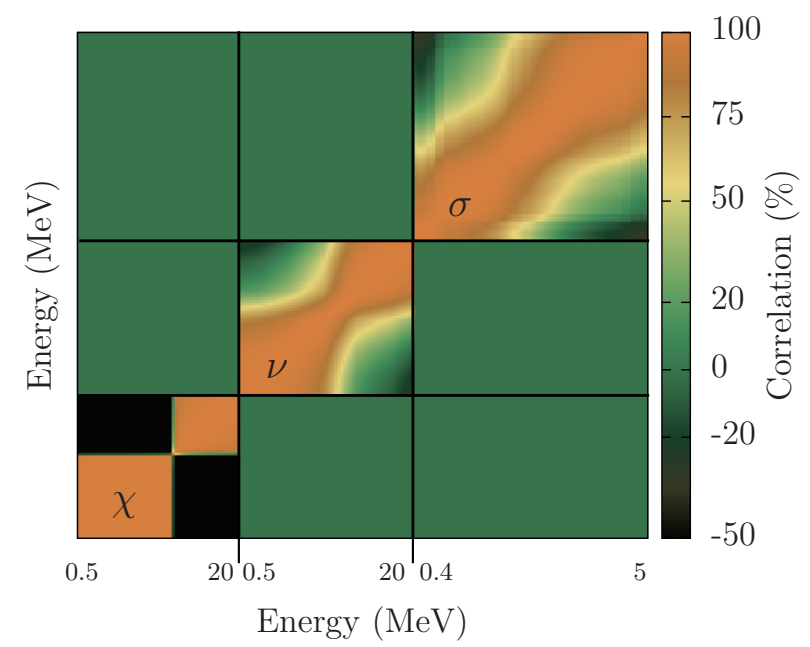

Fig. 2. Prior correlation matrix for ${ }^{239} \mathrm{Pu} v, \sigma$ and $\chi$ (for the incident neutron energy of $750 \mathrm{keV}$ ). The energy axis is for the incident neutrons for $v$ and $\sigma$, and for the outgoing neutron for $\chi$. The $\mathrm{X}$ - and $\mathrm{Y}$-axis are in linear scale.

or pmf benchmarks. The thermal and intermediate benchmarks (plutonium solution thermal and the plutonium metal intermediate) are also very sensitive to ${ }^{239} \mathrm{Pu}$, but mainly below a few tens of $\mathrm{keV}$.

Using the set of equations presented in the previous section, the correlations between the three quantities $v, \sigma$ and $\chi$ can be obtained, given a set of benchmarks. This approach is very similar to the nuclear data evaluation with differential nuclear data. When evaluating for instance the ${ }^{239} \mathrm{Pu}$ fission cross section, measurements of the pointwise or groupwise cross section are considered. Due to the experimental correlation of the considered measurements (for instance over an energy range), the evaluated cross section will also be correlated with itself. Additionally, if the constrains from other differential cross section measurements are considered (such as the total or reaction cross sections), cross-correlations between these different types will be obtained. The method proposed in this paper is therefore the counterpart for integral measurements.

As a consequence, the integral $k_{\text {eff }}$ benchmarks are used in the following to obtain correlation matrices for nuclear data which have high sensitivities. The prior correlations used for ${ }^{239} \mathrm{Pu}$ are presented in Figure 2. Three distinct blocks with non-zero correlations can be seen and correspond to energy-energy correlations for the same quantity. As explained, there are no cross-correlations between these three quantities. Specific patterns are only due to the considered models as no differential experiments are considered (e.g. the strong correlations observed for the $\chi$ matrix comes from the use of the Madland-Nix model, see reference [18] for details).

The correlation matrix between $v$ and $\sigma$ for different incident neutron energies are presented in Figure 3 for a specific benchmark.

As presented, such correlation values are depending on the type of considered benchmarks, namely fast or thermal.

In the case of the fast benchmark, two clear zones are visible with a border at the incident energies of the cross section $\approx 500-600 \mathrm{keV}$. Anti-correlations between $v$ and $\sigma$

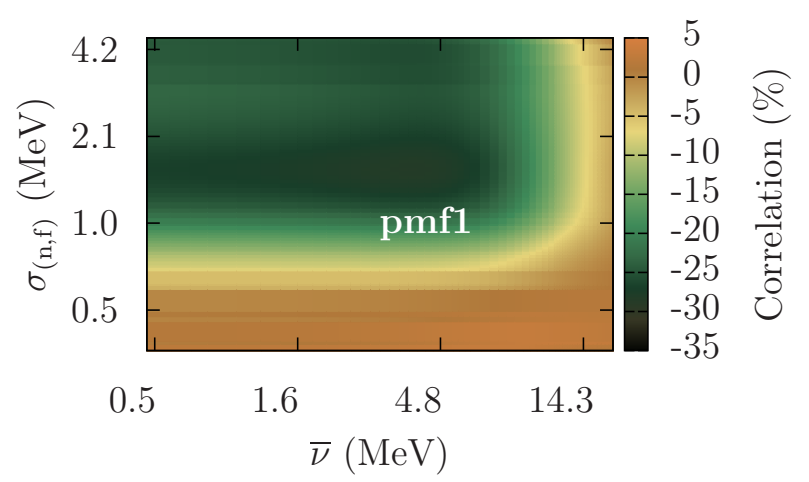

Fig. 3. Correlation matrix between ${ }^{239} \mathrm{Pu} v$ and $\sigma$ considering the fast pmf1 benchmark. The $\mathrm{X}$ - and $\mathrm{Y}$-axis are in log scale.

are expected from the benchmark itself in the energy region where the neutron population is important: a high neutron multiplicity can be compensated by a low fission cross section to obtain a specific $k_{\text {eff }}$. In the energy region where the neutron population is weak, no noticeable correlation is found, even if the correlation is gradually fading away, due to the mentioned internal correlation. The example of this benchmark is representative of the other fast cases.

In a similar way, the correlations between $\chi$ and $v$ can also be obtained. No explicit examples are provided here, as the final correlation matrix is presented in the next section. The impact of the additional anti-correlations can be seen in Table 1 where the prior and posterior $k_{\text {eff }}$ and the calculated uncertainties are presented. The posterior values are calculated using the weights $\omega_{i}$ for both the averages and the uncertainties (standard deviations). The calculated over measured $k_{\text {eff }}$ are also presented as $C / E$ ratios to indicate the impact of the weights compared to the reference (measured) values. For all the considered benchmarks, the calculated uncertainties are strongly reduced (by more than a factor 2 ) and the average $k_{\text {eff }}$ is in better agreement with the experimental value. To illustrate the change in the pdf, Figure 4 presents the prior and posterior distributions for two benchmarks: pmf1 and pmf13. In the case of the pmf1 benchmark, the central value of the prior distribution is already relatively close to the experimental reference. Therefore the posterior average is not drastically changed. On the contrary, the prior uncertainty is large compared to the experimental value, leading to a posterior distribution much narrower. In the case of the pmf13 benchmark, same observation can be done for the uncertainty, but the average value is also strongly changed, as the prior distribution is shifted

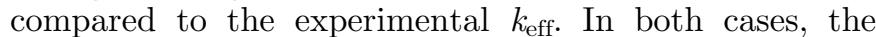
posterior distributions reflect the constraints from the integral data.

\section{Combination}

Based on the previous results, the correlation matrix presented in Figure 2 can be updated for the three considered quantities. With a set of fast benchmarks, the weighted average correlation matrices can be calculated, as it can be done during the usual evaluation process with 
Table 1. Prior and posterior average $k_{\text {eff }}$ and uncertainties for selected benchmarks. Uncertainties $\Delta k$ are given in pcm. $C / E$ values are also indicated.

\begin{tabular}{|c|c|c|c|c|c|c|}
\hline \multirow[t]{2}{*}{ Benchmark } & \multicolumn{2}{|c|}{ Prior } & \multicolumn{2}{|c|}{ Posterior } & \multirow[t]{2}{*}{$C / E-1$ Prior $(\%)$} & \multirow[t]{2}{*}{$C / E-1$ Posterior $(\%)$} \\
\hline & $\bar{k}$ & $\pm \Delta k$ & $\bar{k}$ & $\pm \Delta k$ & & \\
\hline pmf1 & 1.00082 & \pm 782 & 0.99999 & \pm 133 & 0.08 & 0.00 \\
\hline pmf2 & 1.00171 & \pm 705 & 1.00023 & \pm 143 & 0.17 & 0.02 \\
\hline pmf3-1 & 1.00240 & \pm 725 & 1.00016 & \pm 207 & 0.24 & 0.02 \\
\hline pmf5-1 & 1.00056 & \pm 782 & 1.00002 & \pm 93 & 0.06 & 0.02 \\
\hline pmf6-1 & 1.00156 & \pm 700 & 1.00018 & \pm 218 & 0.15 & 0.02 \\
\hline pmf13-1 & 1.00789 & \pm 770 & 1.00356 & \pm 160 & 0.45 & 0.01 \\
\hline pmf35-1 & 0.99755 & \pm 760 & 0.99994 & \pm 113 & 0.25 & 0.01 \\
\hline pmf44-1 & 0.99878 & \pm 695 & 0.99772 & \pm 144 & 0.11 & 0.00 \\
\hline pmi2-1 & 1.01766 & \pm 1018 & 0.98788 & \pm 209 & 3.11 & 0.10 \\
\hline
\end{tabular}
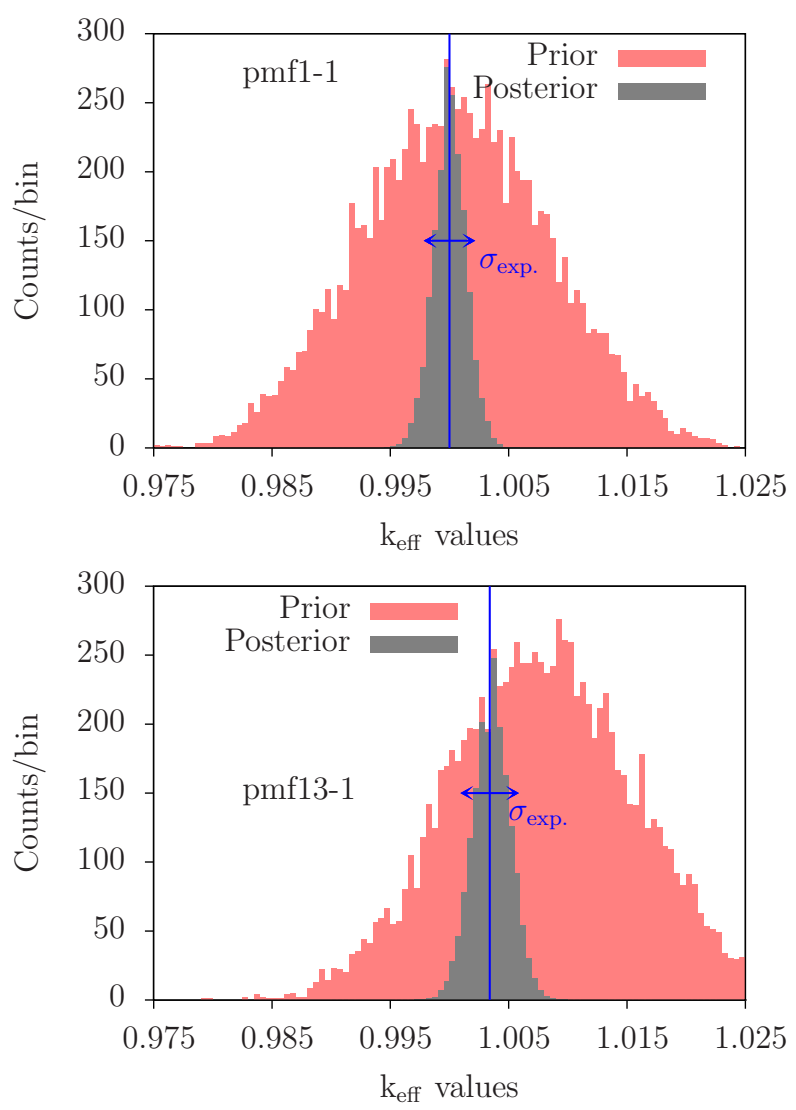

Fig. 4. Prior and posterior distributions of $k_{\text {eff }}$ for two benchmarks: pmf1 and pmf13. The blue line indicate the experimental value with its uncertainty.

differential data (the weight is driven here by the experimental uncertainty of $k_{\text {eff }}$, but any other definition can be used). Considering 9 fast benchmarks (pmf1, pmf3, pmf9, pmf10, pmf19, pmf20, pmf24, pmf35 and pmf44, only the first configuration for each of these benchmarks), the posterior correlation matrix is calculated and presented in Figure 5.

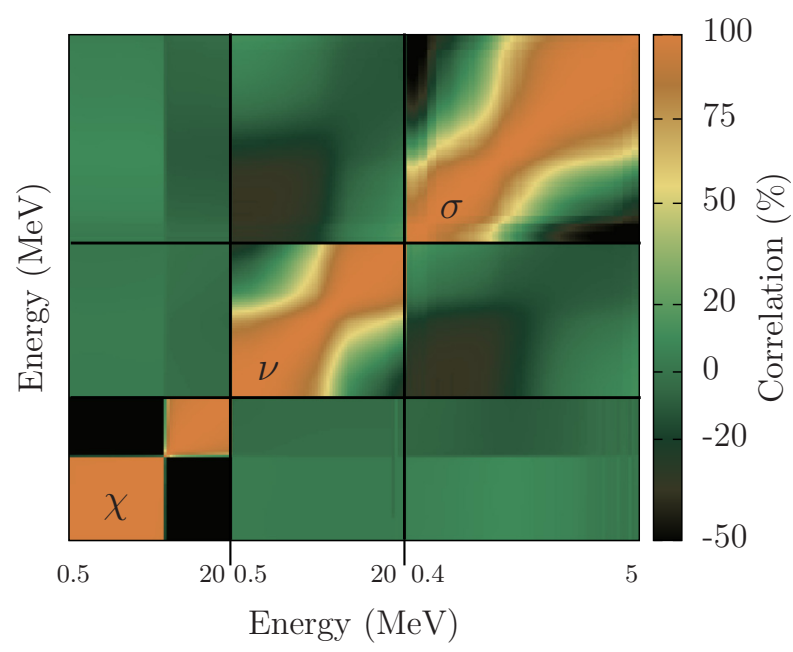

Fig. 5. Posterior correlation matrix for ${ }^{239} \mathrm{Pu} v, \sigma$ and $\chi$ (for the incident neutron energy of $750 \mathrm{keV}$ ). The energy axis is for the incident neutrons for $v$ and $\sigma$, and for the outgoing neutron for $\chi$. The $\mathrm{X}$ - and $\mathrm{Y}$-axis are in linear scale.

The number of 9 benchmarks is certainly not enough to achieve a satisfactory convergence of the average correlation factors, but similarly to the evaluation of the cross sections, the number of experimental data is often limited. Nuclear data evaluators frequently consider such a small number of data; in the case of the maximum correlation, the $95 \%$ confidence interval is obtained within a \pm 0.16 correlation band. As mentioned in the previous section, non-zero cross-correlation terms are observed. The strongest cross-correlation links the fission cross section and the neutron multiplicity, which globally remain negative for all incident neutron energies. The cross-correlation for the two other quantities are weaker, although not zero. In the context of a full evaluation for the inclusion in a specific library, a variety of sensitive benchmarks needs to be used. Also, the thermal and resonance energy range needs to be studied, with dedicated thermal and intermediate benchmarks. The present study nevertheless demonstrates the 
possibility of using integral values to calculate correlations which are not available otherwise. The observed negative correlation between the fission cross section and the neutron multiplicity will certainly impact the calculated uncertainties for various applications, for instance in reactor calculations. Alternatively, such method can be used to obtain correlation matrices between different isotopes. In the case of ${ }^{238} \mathrm{U}$ and ${ }^{235} \mathrm{U}$, many of their nuclear data affect integral observables. An integral benchmarks with an intermediate ${ }^{235} \mathrm{U}$ enrichment, such as the "Big-10 (or imf7 for intermediate-metal-fast benchmark)" will certainly lead to non-negligible cross-correlations. An other method can be used following the work presented in reference [14]: the effect of nuclear data is observed on the simulation of realistic reactor core cycle operations. Such simulation can be compared to in-core measurements and thus replacing the $k_{\text {eff }}$ quantity used in this study.

It can be noticed that the update of the uncertainties is not proposed in this work, but only the (cross-)correlations. The use of integral experiments for the reduction of uncertainties for differential quantities is a subject of discussion and no consensus is yet achieved. Compensating factors can play an important role and might not be applicable in all cases for a general-purpose library. It is different for correlation matrices: they are already implicitly included in evaluations by not being present. Missing correlations are equal to zero correlation, and if not entered in a library, they can be considered as shadow correlations. The proposed method merely offers an alternative to turn them into explicit quantities (note that a consequence of modifying correlation factors can be a re-evaluation of the uncertainties).

\section{Conclusion}

Cross-correlation matrices between the fission cross section, the neutron multiplicity and their spectra were calculated based on fast integral experiments. The integration of the criticality benchmarks follows a classical Bayesian approach. It is not intended to replace the traditional evaluation methods based on differential measurements, but the use of integral quantities can provide cross-correlations for nuclear data apparently not connected by the reaction models or by differential experiments. We would like to remind that missing correlations are equivalent to zero correlations. If not explicitly given, these zero correlations are shadow correlations which affect calculated quantities. Therefore, the proposed method turns hidden correlations into actual information. As mentioned, it can be extended to quantify cross-isotope correlations, as between ${ }^{235} \mathrm{U}$ and ${ }^{238} \mathrm{U}$ and finally, be part of a general evaluation process for a nuclear data library based on differential and integral constraints.

\section{Perspectives}

One of the goals of such work is to complement the covariance information provided in nuclear data libraries. The present example does not represent an approach which is general enough for the insertion in such library, but it is one step towards this direction. In this context, the following remarks can be mentioned:

- the example of $\sigma, v$ and $\chi$ can be extended to other nuclear data quantities. Other cross sections, such as capture, will be positively correlated with fission and other ones using integral benchmarks. For the thermal and resonance range, correlations can also be extracted using a multigroup representation for the cross sections since the energy grid is extremely dense;

- such correlation factors are complementary to the ones obtained from differential measurements and theoretical calculations of differential quantities. They are not contradictory and should be used together for a final evaluation, for instance using a set of differential and integral weights (as presented in reference [31]);

- for a complete evaluation using the integral information, a variety of quantities needs to be involved, covering both a large energy region and divers integral quantities (not only $k_{\text {eff }}$, but also reaction rates, spectra indexes, activation, or emitted neutron spectra from pulsed spheres. Additionally, the correlations between benchmarks need to be taken into account, for instance using a generalized chi-2 instead of equation (1). In this case, the experimental covariance matrices need to be assessed with care since their impact will be of importance;

- as mentioned, integral benchmarks can also bring correlations between isotopes (e.g. Big-10 $k_{\text {eff }}$ for correlations between ${ }^{235} \mathrm{U}$ and ${ }^{238} \mathrm{U}$, but also between various reactions using spectra indexes);

- the current ENDF-6 format used for the nuclear data evaluation does not allow for correlation matrices between quantities such as $\sigma$ and $v$. An update of this format is therefore necessary;

- in the context of a full evaluation of covariance matrices, it is also important to provide "statistically converged" correlations. It is therefore necessary to use as many integral experiments as necessary to obtain correlation values which do not changed outside a given limit, when adding new experiments. Again, this problematic is very similar to the case of differential data.

The combination of the integral and differential correlations can be complemented by the adjustment of the variances, taking into account both sources of information. This would lead to fully updated covariance matrices, following a well defined and reproducible scheme. Such work would then be part of the elaboration of a nuclear data library based on models (for differential data), realistic model parameter distributions and integral constraints, as presented in reference [33].

\section{References}

1. P. Romojaro, F. Alvarez-Velarde, I. Kodeli, A. Stankovskiy, C.J. Diez, O. Cabellos, N. Garcia-Herranz, J. Heyse, P. Schillebeeckx, G. Van den Eynde, G. Zerovnik, Nuclear data sensitivity and uncertainty analysis of effective neutron multiplication factor in various MYRRHA core configurations, Ann. Nucl. Energy 110, 330 (2017) 
2. M. Aufiero, M. Martin, M. Fratoni, XGPT: extending Monte Carlo generalized perturbation theory capabilities to continuous-energy sensitivity functions, Ann. Nucl. Energy 96, 295 (2016)

3. D. Rochman, A. Vasiliev, H. Ferroukhi, T. Zhu, S.C. van der Marck, A.J. Koning, Nuclear data uncertainty for criticalitysafety: Monte Carlo vs. linear perturbation, Ann. Nucl. Energy 92, 150 (2016)

4. M. Pusa, Incorporating sensitivity and uncertainty analysis to a lattice physics code with application to CASMO-4, Ann. Nucl. Energy 40, 153 (2012)

5. W. Zwermann, A. Aures, L. Gallner, V. Hannstein, B. Krzykacz-Hausmann, K. Velkov, J.S. Martinez, Nuclear data uncertainty and sensitivity analysis with XSUSA for fuel assembly depletion calculations, Nucl. Eng. Technol. 46, 343 (2014)

6. M.L. Williams, B.T. Rearden, SCALE-6 Sensitivity/ uncertainty methods and covariance data, Nucl. Data Sheets 109, 2796 (2008)

7. A. Hoefer, O. Buss, M. Hennebach, M. Schmid, P. Porsch, MOCABA: a general Monte Carlo-Bayes procedure for improved predictions of integral functions of nuclear data, Ann. Nucl. Energy 77, 514 (2015)

8. C.J. Diez, O. Cabellos, D. Rochman, A.J. Koning, J.S. Martinez, Monte Carlo uncertainty propagation approaches in ADS burn-up calculations, Ann. Nucl. Energy 54, 27 (2013)

9. N. Garcia-Herranz, O. Cabellos, J. Sanz, J. Juan, J.C. Kuijper, Propagation of statistical and nuclear data uncertainties in Monte Carlo burn-up calculations, Ann. Nucl. Energy 35, 714 (2008)

10. E. Alhassan, H. Sjostrand, P. Helgesson, M. Osterlund, S. Pomp, A.J. Koning, D. Rochman, On the use of integral experiments for uncertainty reduction of reactor macroscopic parameters within the TMC methodology, Prog. Nucl. Energy 88, 43 (2016)

11. D. Rochman, O. Leray, A. Vasiliev, H. Ferroukhi, A.J. Koning, M. Fleming, J.C. Sublet, A Bayesian Monte Carlo method for fission yield covariance information, Ann. Nucl. Energy 95, 125 (2016)

12. P. Helgesson, D. Rochman, H. Sjostrand, E. Alhassan, A.J. Koning, $\mathrm{UO}_{2}$ versus $\mathrm{MOX}$ : propagated nuclear data uncertainty for $k_{\text {eff }}$, with burnup, Nucl. Sci. Eng. 177, 321 (2014)

13. D. Rochman, O. Leray, M. Hursin, H. Ferroukhi, A. Vasiliev, A. Aures, F. Bostelmann, W. Zwermann, O. Cabellos, C.J. Diez, J. Dyrda, N. Garcia-Herranz, E. Castro, S. van der Marck, H. Sjostrand, A. Hernandez, M. Fleming, J.-Ch. Sublet, L. Fiorito, Nuclear data uncertainties for typical LWR fuel assemblies and a simple reactor core, Nucl. Data Sheets 139, 1 (2017)

14. O. Leray, H. Ferroukhi, M. Hursin, A. Vasiliev, D. Rochman, Methodology for core analysis with nuclear data uncertainty quantification and application to Swiss PWR operated cycles, submitted to Annals of Nuclear Energy, December 2016

15. M.B. Chadwick, P. Oblozinsky, M. Herman, N.M. Greene, R.D. McKnight, D.L. Smith, P.G. Young, R.E. MacFarlane, G.M. Hale, S.C. Frankle, A.C. Kahler, T. Kawano, R.C. Little, D.G. Madland, P. Moller, R.D. Mosteller, P.R. Page, P. Talou, H. Trellue, M.C. White, W.B. Wilson, R. Arcilla, C.L. Dunford, S.F. Mughabghab, B. Pritychenko, D. Rochman, A.A. Sonzogni, C.R. Lubitz, T.H. Trumbull, J.P. Weinman, D.A. Brown, D.E. Cullen, D.P. Heinrichs, D.P.
McNabb, H. Derrien, M.E. Dunn, N.M. Larson, L.C. Leal, A.D. Carlson, R.C. Block, J.B. Briggs, E.T. Cheng, H.C. Huria, M.L. Zerkle, K.S. Kozier, A. Courcelle, V. Pronyaev, S.C. van der Marck, ENDF/B-VII.0: Next generation evaluated nuclear data library for nuclear science and technology, Nucl. Data Sheets 107, 2931 (2006)

16. M. Chadwick, M. Herman, P. Oblozinsky, M.E. Dunn, Y. Danon, A.C. Kahler, D.L. Smith, B. Pritychenko, G. Arbanas, R. Arcilla, R. Brewer, D.A. Brown, R. Capote, A.D. Carlson, Y.S. Cho, H. Derrien, K. Guber, G.M. Hale, S. Hoblit, S. Holloway, T.D. Johnson, T. Kawano, B.C. Kiedrowski, H. Kim, S. Kunieda, N.M. Larson, L. Leal, J.P. Lestone, R.C. Little, E.A. McCutchan, R.E. MacFarlane, M. MacInnes, C.M. Mattoon, R.D. McKnight, S.F. Mughabghab, G.P.A. Nobre, G. Palmiotti, A. Palumbo, M.T. Pigni, V.G. Pronyaev, R.O. Sayer, A.A. Sonzogni, N.C. Summers, P. Talou, I.J. Thompson, A. Trkov, R.L. Vogt, S.C. van der Marck, A. Wallner, M.C. White, D. Wiarda, P.G. Young, ENDF/B-VII.1 Nuclear data for science and technology: cross sections, covariances, fission product yields and decay data, Nucl. Data Sheets 112, 2887 (2011)

17. K. Shibata, O. Iwamoto, T. Nagagawa, N. Iwamoto, A. Ichihara, S. Kunieda, S. Chiba, K. Furutaka, N. Otuka, T. Ohasawa, T. Murata, H. Matsunobu, A. Zukeran, S. Kamada, J. Katakura, JENDL-4.0: a new library for nuclear science and engineering, J. Nucl. Sci. Technol. 48, 1 (2011)

18. A.J. Koning, D. Rochman, Modern nuclear data evaluation with the TALYS code system, Nucl. Data Sheets 113, 2841 (2012)

19. A.J. Koning, D. Rochman, Towards sustainable nuclear energy: putting nuclear physics to work, Ann. Nucl. Energy 35, 2024 (2008)

20. E. Bauge, P. Dossantos-Uzarralde, Evaluation of the covariance matrix of ${ }^{239} \mathrm{Pu}$ neutronic cross sections in the continuum using the backward-forward Monte-Carlo method, J. Korean Phys. Soc. 59, 1218 (2011)

21. H.Y. Lee, R.C. Haight, T.A. Bredeweg, M. Devlin, N. Fotiades, M. Jandel, A. Laptev, R.O. Nelson, J.M. O'Donnell, B.A. Perdue, T.N. Taddeucci, J.L. Ullmann, S.A. Wender, M.C. White, C.Y. Wu, A. Chyzr, R.A. Henderson, E. Kwan, Prompt fission neutron spectrum study at LANSCE: CHI-NU project, in Proceedings of the Fifth International Conference on ICFN5, Sanibel Island, Florida, USA, 4-10 November 2012 (2012)

22. H. Leeb, St. Gundacker, D. Neudecker, V. Wildpaner, The GENEUS Project - development of an evaluation tool, J. Korean Phys. Soc. 59, 1230 (2011)

23. R. Capote, D.L. Smith, An investigation of the performance of the unified Monte Carlo method of neutron cross section data evaluation, Nucl. Data Sheets 109, 2725 (2008)

24. R. Capote, D. Smith, A. Trkov, M. Meghzifene, A new formulation of the Unified Monte Carlo approach (UMC-B), and cross-section evaluation for the dosimetry reaction ${ }^{55} \mathrm{Mn}$ $(\mathrm{n}, \gamma)^{56} \mathrm{Mn}$, J. ASTM Int. 9, JAI104115 (2012)

25. A.J. Koning, Bayesian Monte Carlo method for nuclear data evaluation, Eur. Phys. J. A 51, 184 (2015)

26. D. Rochman, A.J. Koning, S.C. van der Marck, Improving neutronics simulations and uncertainties via a selection of nuclear data, Eur. Phys. J. A 51, 182 (2015)

27. J.B. Briggs Ed., International Handbook of evaluated Criticality Safety Benchmark Experiments, NEA/NSC/ DOC(95)03/I (Organisation for Economic Co-operation and Development, Nuclear Energy Agency, 2004) 
28. T. Goorley, MCNP 6.1.1-Beta Release Notes, Los Alamos National Laboratory, Report LA-UR-14-24680, June 2014

29. A.J. Koning, D. Rochman, J. Kopecky, J. Ch. Sublet, E. Bauge, S. Hilaire, P. Romain, B. Morillon, H. Duarte, S. van der Marck, S. Pomp, H. Sjostrand, R. Forrest, H. Henriksson, O. Cabellos, S. Goriely, J. Leppanen, H. Leeb, A. Plompen, R. Mills, TENDL-2014: TALYS-based evaluated nuclear data library (2014), ftp://ftp.nrg.eu/pub/www/talys/ tendl2014/tendl2014.html
30. T. Zhu, A. Vasiliev, H. Ferroukhi, D. Rochman, A. Pautz, Testing the sampling-based NUSS-RF tool for the nuclear data related global sensitivity analysis with Monte Carlo neutronics calculations, Nucl. Sci. Eng. 69, 184 (2016)

31. D. Rochman, A.J. Koning, Evaluation and adjustment of the neutron-induced reactions of ${ }^{63,65} \mathrm{Cu}$, Nucl. Sci. Eng. 170, 265 (2012)

32. A. Vasiliev, D. Rochman, M. Pecchia, H. Ferroukhi, Energies 9, 1039 (2016)

33. E. Bauge, M. Dupuis, S. Hilaire, S. P $\backslash$ 'eru, A.J. Koning, D. Rochman, S. Goriely, Nucl. Data Sheets 118, 32 (2014)

Cite this article as: Dimitri Rochman, Eric Bauge, Alexander Vasiliev, Hakim Ferroukhi, Correlation $\bar{v}_{p}-\sigma-\chi$ in the fast neutron range via integral information, EPJ Nuclear Sci. Technol. 3, 14 (2017) 